\title{
A New Technique of Core Muscle Strength Training for Hurdlers
}

\author{
WEN Bo ${ }^{1 *}$ and WEN TianYang ${ }^{2}$ \\ ${ }^{1}$ Jieyang college, Guangdong Jieyang, china \\ ${ }^{2}$ University of macau, university of commerce and industry, china \\ *Corresponding author: WEN Bo, jieyang college, Guangdong Jieyang, china
}

\section{ARTICLE INFO}

Received: 幽 June 26, 2019

Published: 幽 July 02, 2019

Citation: WEN Bo, WEN TianYang. A New Technique of Core Muscle Strength Training for Hurdlers. Biomed J Sci \& Tech Res 19(2)-2019. BJSTR. MS.ID.003284.

\begin{abstract}
In this paper. To study the best speed of hurdler's fast hurdling technique, the method of analogy analysis is adopted by consulting data. Using the core muscle strength method to train hurdlers to run fast combined with the efficiency of hurdling technology, this paper analyzes the method of improving speed, and concludes that the efficiency method of improving hurdlers' attacking speed function is obtained. Innovate hurdling training thinking, reduce hurdling speed loss, and discover a new concept of hurdling speed training.
\end{abstract}

Keywords: Short Distance Hurdles; Hurdling Step; Hurdle Speed; Core Strength Training

\section{The Mechanism of Core Muscle Strength Training}

\section{Hurdler's Fast Hurdling Technique Rate is Analyzed \\ Hurdles movement}

Hurdles movement is a hip rotation frequency and the most rapid severe track and field events, the hurdles is speed sports project, there are 10 hurdles step, every hurdle requirement hurdler tapping column leg swing bar, leg press, the take-off leg ground lift column shear Angle of folding, factors influencing the hurdler technology more. In the past, the traditional strength training and hurdling technique are not appropriate, and the improving speed is not ideal. Hurdlers are crucial to the absolute speed requirement. Absolute speed is greatly supported by the core muscle strength, which determines the stable support points for hurdlers to push back, swing forward, fold and scrape the ground, thus generating a powerful source of power. Core muscle strength is related to the waist hip flexion, stretching hip hip to exercise of power, attack down the conduction knee joint after the bar, produce bend flexion, waste of work, involving the onset of excessive torque speed hurdler body loss, lack of core muscle strength hip flexion and hip fatigue, stress transfer lower limb muscles, deep knee bends, joint muscle strength offset bar work speed, hurdle is not easy and effortless.

Lumbar core muscle strength size effect stretching hip flexible rotation speed, affect the coordination of two arms swinging, losses run speed, strong core muscle strength can make run leg lifts no back, strong core muscle strength can make folding the ground movements back and strong core muscle strength can be prompted to run smoothly and shaking, stable control center of gravity acceleration and strong core muscle strength can automatically control the hurdler run bar speed rhythm, strong core muscle strength can cross bar or column leg down quickly, quickly completed across from the bar, can perfect joining ran fast attack bar technology accurately.

\section{Core Muscle Strength}

Core muscle strength is applied to track and field training, to explore and study the mechanism of core muscle strength training for hurdlers' fast attack speed technical function, so that the attack speed will not be lost and the fast hurdle technique will be improved. It is believed that core balance training can enhance the control ability of hurdlers under the unsteady state, maintain the balance function, coordinate the strength performance of each muscle group, improve the hurdlers' skill and prevent sports injury. The core control ability of hurdler's body is the main factor of the movement function of upper and lower limbs. Strengthening the core muscle strength is beneficial to control the center of gravity, determine the fulcrum for the movement of upper and lower limbs, and optimize the transmission and control of the generated force. The core parts include spine, hip joint, upper and lower limbs, which play a pivotal role in maintaining balance in the physical movement 
of hurdlers. Core balance depends on the control and contraction of the trunk core muscle group. The core part of hurdler's body generates the source of speed and power, transmission and control for hurdler [1].

\section{Body Core}

Body core shows core muscle group speed and physical quality of hurdlers are all controlled by the nervous system. The central nervous system functions hurdlers' muscles drives muscle strength and controls muscle group, so that core balance reflects dynamic sense. Hurdler core body balance depends on the core muscle strength, the coordination between the trunk of each joint muscles to complete the hurdler body, for the onset of muscle contraction parts to determine the power protection, increase the efficiency of the onset of muscle and association each muscle contraction, speed up the transmission power, improve the efficiency of hurdler body movement. Core balance is the timer of physical movement of hurdlers, which plays a regulating role in training to stabilize the body movement of hurdlers, balance and coordinate the physical function of hurdlers. Core muscle strength training can assist hurdler core stability, strong core muscle strength can be stable hurdler core parts of the body, control hurdler body movement center of gravity, passing the powers of their lower limbs, speed, balance the stability and fixation of further smaller muscle groups, complete the speed of the neural control muscle strong ability, as a hurdle sports power source, the core muscle strength support to control the hurdler rotating body gravity balance, power drag, improve the core power conversion is hurdler hurdles speed engine [2].

\section{Core Muscle}

Core muscle strength training for hurdle athletes run hurdles, increase muscle strength as the core torso motion together, build support for onset of muscle contraction, the muscle contraction force, coordinate various hurdles joint muscle into the state, make the different movement joints, muscle contraction joint movement, makes the muscle rotation of each joint, coordination between muscle movement, strengthen the core parts of the lumbar spine and hip muscles, enhance the overall strength of the core muscles passing ability. Resistance strength training can improve the coordination of multiple muscle groups, the rapid recruitment of synergistic fast muscle and nerve impulse frequency [3]. Increase the muscle contraction strength of upper and lower limbs, rapidly transfer strength, improve signal transmission, improve the efficiency of hurdlers' special hurdling exercise, and create conditions for hurdlers' fast running muscle contraction work.

\section{During Hurdles}

During hurdles, hip rotation, accompanied by weak muscle strength and muscle tension disorder of lumbar segments, affects the balance of lumbar spine, and strengthens core muscle strength training measures of hurdlers. The trunk muscles of a hurdler from the middle to the legs, directly behind and on both sides, can regulate the core muscles of the hurdler's body to maintain the balance and stability of the trunk. The control of trunk core muscle group will effectively control the ability of balanced movement over hurdles. In sports, hurdlers lack the support of large muscle groups in their lumbar vertebrae, while hurdlers are in the unsupported condition when hurdling, which is the weak part in sports. Core muscle strength training is needed to protect the movement center to enhance balance. At present, the hurdle project mainly focuses on the training of lower limbs and legs, but the training of core muscle strength is not enough, and there are not enough methods to train hurdle.

After 1.6 run-up hurdles through hurdler body back muscles to integration, the hurdler body hurdles with core muscle strength exercise on practice, to improve motor function and its mechanism is through the hurdler body core muscle strength training of the nervous system control of the waist muscle, strengthen the hurdles small muscles and big muscles strength coordination function, enhance the speed signal input, upward lower limb muscle signals, to promote coordinated movement, all joints, muscles training can enhance the body of the contralateral limb speed sensitivity, through the training can accelerate the brain function for the hurdler leg control ability, The adjustment response of core motor muscle group is closely related to the physical balance and control ability of hurdlers, and the nervous system should have fine ability to control and control multiple muscles [4].

\section{Through the Core Muscle Strength Training}

Through the core muscle strength training, can be induced by central nervous of hurdles movements to make various regulation, make the hurdler body movement direction of upper and lower limbs movement direction on the dynamic were four line, ensure the hurdler body body and hurdling action form rapid transmission, promote the hurdler ran fast function, core muscle group source dominate bilateral pyramidal tract of nervous system, promote the hurdler normal function. Hurdler core body muscles contraction of determine the fulcrum for hurdles limb muscle power, and increase the efficiency of the hurdles limb muscle contraction and coordinate each joint movement, the transfer function of limbs strength hurdles, core balance training can improve the hurdles body system coordination control ability, make the hurdler can give full play to their strength.

\section{The Hurdler Shall Determine the Stable Working Fulcrum}

To prevent sports injury through strength training, to build stable fulcrum for hurdlers to do work by coordinating the core parts of hurdlers' bodies, and to reduce the negative pressure on the end of limbs muscles; Strength exercises can enhance the function of spinal adjustment and protection, and the strength of core muscles determines the size of spinal function. Hurdler core muscle strength exercise effectively promote nervous system 
around quickly raise lumbar muscles, nerve impulse frequency coordination coordinated movements more muscle groups, common activated spinal muscle coordination, improve the lumbar stretch flexor muscle function of neuromodulation sensitivity, activate the nerve input speed fast, and impulse response time was shortened, and the synchronization control each muscle group ability, improve the lumbar muscles contraction force. The central nervous system regulates the coordination of the extensor and flexor muscles to keep the lumbar spine stable. The mechanism of lumbar abdominal muscle group load distribution is not perfect, and the motor muscle neurons are sensitive to input, which can improve the load pressure balance state, the sensitivity of muscle position, and maintain the dynamic stability of the lumbar position, especially the core muscle strength training [5].

\section{The Training is Dynamic and Stable for Hurdlers}

To improve the balance of the hurdler's waist and improve the motion sensitivity of the interlumbar joints; Improve lumbar intervertebral joint movement precision work; Rely on the lumbar muscles to continue the movement; To improve the efficiency of lumbar nervous system recruitment and contraction. Core balance is dynamic stability, lumbar extensor flexor dynamic control joint exercise to improve balance. It is the basis of core balance and core muscle strength training to change the original structure of the fast tapping speed, so as to avoid positioning errors in the dynamic stable movement tissue and improve the accuracy of lumbar lumbar extensor and flexor muscle repositioning. Can reduce speed loss after hurdle effect.

\section{High-Intensity Muscle Strength Training Can Temporarily Change the Blood Pressure of Hurdlers}

The mechanism is that training can improve the regulating function of the central nervous system and adjust the imbalance between endocrine imbalance and vegetative nervous function. Brain center of sympathetic nerve activity, make the blood of

\section{ISSN: 2574-1241}

DOI: 10.26717/BJSTR.2019.19.003284

WEN Bo. Biomed J Sci \& Tech Res

This work is licensed under Creative Commons Attribution 4.0 License

Submission Link: https://biomedres.us/submit-manuscript.php norepinephrine, epinephrine, causing vascular contractility of the active material is reduced, the blood caused by histamine, stimulating peptide vasodilation of active substances, in the whole body muscle relaxation causes blood vessels to relax the cooperation with the reflective rotate open capillary network, the blood capillary expansion, microcirculation increased, thereby reducing the blood vessels, total peripheral resistance, causing blood pressure to drop. Other factors include human hormones and plasma renin, angiotensin II (Ang II ) and aldosterone levels drop, sensitivity and lower beta receptors, by neurohumoral mechanisms regulating effective blood volume ability is poor, prone to low blood volume sex low blood pressure (hypotension) [6].

\section{Conclusion}

The core muscle strength training of hurdlers' fast attack is different from that of competitive sports. A scientific and reasonable training plan is made to track the function, function and shape of hurdlers after training. The function of hurdlers after training, using core muscle strength to train hurdlers to make regular functional evaluation in time, analyze the adjustment plan of training content, modify hurdlers' function in time, innovate ideas, and transform into the best sports technical state of hurdlers with the best practice effect.

\section{References}

1. Xiao ping Chen (2007) Training of core stability forces. Sports science 9: 2 .

2. Yun long deng (2007) sports training of thinking and design. Beijing China education culture press p. 16-65.

3. house xing (1986) sports training, sports press. Beijing 1986-1978.

4. Liu aijie, li shaodan (2007) Core training of competitive sports. Chinese sports coaches 45(4): 2-6.

5. Feng jianjun, yuan jianguo (2009) Research review on core stability and core strength. Journal of physical education 11: 015.

6. Yao Songping (2006) Inherit and carry forward the matt d also professor's academic thoughts in sports training. sports science p. 11. 